\title{
Is there an association between female gender and outcome in severe trauma? A multi-center analysis in the Netherlands
}

\author{
M. Pape ${ }^{1 *}$ D, G. F. Giannakópoulos ${ }^{1}$, W. P. Zuidema ${ }^{1}$, E. S. M. de Lange-Klerk², E. J. Toor ${ }^{3}$, M. J. R. Edwards ${ }^{4}$,
} M. H. J. Verhofstad ${ }^{5}$, T. N. Tromp ${ }^{4}$, E. M. M. van Lieshout ${ }^{6}$, F. W. Bloemers ${ }^{1}$ and L. M. G. Geeraedts ${ }^{1}$

\begin{abstract}
Introduction: Little evidence suggest that female gender is associated with a lower risk of mortality in severely injured patients, especially in premenopausal women. Previous clinical studies have shown contradictory results regarding protective effects of gender on outcome after severe trauma. The objective of this study was to determine the association between gender and outcome (mortality and Intensive Care Unit (ICU) admission) among severely injured patients in the Netherlands.
\end{abstract}

Methods: A retrospective multicentre study was performed including all polytrauma patients (Injury Severity Score $(I S S) \geq 16)$ admitted to the ED of three level 1 trauma centres, between January 1st, 2006 and December 31 st, 2014. Data on age, gender, mechanism of injury, ISS, Abbreviated Injury Scale (AIS), prehospital intubation, Revised Trauma Score (RTS), systolic blood pressure (SBP) and Glasgow Coma Scale (GCS) upon admission at the Emergency Department was collected from three Regional Trauma Registries. To determine whether gender was an independent predictor of mortality and ICU admission, logistic regression analysis was performed.

Results: Among 6865 trauma patients, male patients had a significantly higher ISS compared to female patients $(26.3 \pm 10.2$ vs $25.3 \pm 9.7, P=<0.0001)$. Blunt trauma was significantly more common in the female group (95.2\% vs $92.3 \%, P=<0.0001)$. Males aged 16- to 44-years had a significant higher in-hospital mortality rate (10.4\% vs $13.4 \%$, $P=0.046)$. ICU admission rate was significantly lower in females $(49.3 \%$ vs $54.5 \%, P=<0.0001)$. In the overall group, logistic regression did not show gender as an independent predictor for in-hospital mortality (OR $1.020(95 \% \mathrm{Cl}$ 0.865-1.204), $P=0.811$ ) or mortality within $24 \mathrm{~h}$ (OR 1.049 (95\% Cl 0.829-1.327), $P=0.693)$. However, male gender was associated with an increased likelihood for ICU admission in the overall group (OR 1.205 (95\% Cl 1.046-1.388), $P=0.010)$.

Conclusion: The current study shows that in this population of severely injured patients, female sex is associated with a lower in-hospital mortality rate among those aged 16- to 44-years. Furthermore, female sex is independently associated with an overall decreased likelihood for ICU admission. More research is needed to examine the physiologic background of this protective effect of female sex in severe trauma.

Keywords: Gender dimorphism, Trauma, Severe injury, Female gender, Mortality, ICU admission

\footnotetext{
* Correspondence: m.pape@vumc.nl

${ }^{1}$ Department of Trauma Surgery, VU University Medical Center, De Boelelaan

1117, P.O. Box 7057, 1007, MB, Amsterdam, The Netherlands

Full list of author information is available at the end of the article
}

(c) The Author(s). 2019 Open Access This article is distributed under the terms of the Creative Commons Attribution 4.0 International License (http://creativecommons.org/licenses/by/4.0/) which permits unrestricted use, distribution, and reproduction in any medium, provided you give appropriate credit to the original author(s) and the source, provide a link to the Creative Commons license, and indicate if changes were made. The Creative Commons Public Domain Dedication waiver (http://creativecommons.org/publicdomain/zero/1.0/) applies to the data made available in this article, unless otherwise stated. 


\section{Introduction}

The effect of gender in severely injured patients has been investigated for many years [1-7]. It is well known that severe trauma results in an inflammatory response with an aberrant production of cytokines by macrophages, resulting in suppression of the immune system [1]. This response is potentially gender dimorphic, for it has been known that cells of the immune system can synthesize sex steroids [1]. Several laboratory studies in rodents have demonstrated that female gender is protective after major trauma, hemorrhage and sepsis [2-4]. Despite the substantial experimental evidence, previous clinical studies have been unable to produce results resembling the laboratory findings $[2,6-8,10-12,14-16]$.

In a German study with 36,000 severely injured patients it was shown that gender alone offers no survival advantage after blunt trauma [5]. However, male gender was an independent negative predictor for morbidity [5]. In another study, no gender-based differences in mortality were shown, but it was concluded that age and ISS were the most important predictors of mortality [13]. In contrast, a large multicenter study demonstrated that severely injured men with an ISS equal to or above 25 and aged 50-years or younger, had a $27 \%$ increased likelihood of dying, compared with women [11]. Similarly, a retrospective Chinese cohort study showed that females had a lower risk of in-hospital mortality compared with males, especially among those 50-years or younger, with an ISS equal to 25 or higher [7]. These studies suggest that a potentially protective effect of female gender is most apparent during the reproductive phase $[7,11]$. Furthermore, they speculate that minor trauma does not lead to immunologic changes and therefore genderbased differences might be more obvious in the severely injured [7].

In an attempt to further elucidate this ongoing controversy, a large retrospective multicenter study was performed to determine the association between gender and outcome, among severely injured patients, admitted at three Level 1 trauma centers in the Netherlands. We hypothesized that female polytrauma patients show decreased mortality rates and lower ICU admission rates.

\section{Patients and methods}

\section{Study subjects}

Patients aged 16 years or older and with an Injury Severity Score (ISS) $\geq 16$ admitted to the Emergency Department (ED) between January 1st, 2006 and December 31st, 2014 were selected for inclusion. Patients who were dead upon arrival at the ED or presented after drowning, electrocution, or strangulation, were excluded. Patients were categorized into three age groups (i.e. 16-44, 45-54, and 55+), to consider the influence of hormonal status attributable to the premenopausal, perimenopausal, and postmenopausal phases. We varied this age threshold for defining the groups, ultimately leading to above mentioned groups. Because females between 16- and 44-years of age are considered hormonally active, a subgroup analysis was performed. Data were retrospectively collected from the regional trauma registries at three Level I trauma centers. These centers were the VU University Medical Center in Amsterdam, the Radboud University medical center in Nijmegen and the Erasmus university medical center in Rotterdam, all located in the Netherlands. The study was approved by the independent Medical Research Ethics Committees of all three hospitals.

\section{Study parameters}

Demographic, injury and outcome parameters for each male and female patient were collected from the trauma registry. The injuries were classified by the Abbreviated Injury Scale (AIS) and the ISS [17, 18]. The Revised Trauma Score (RTS) was used to estimate the severity of the injury and dichotomized (12 or less yes/no). The ISS was used, both as a continuous variable, as well as a categorical variable (16-24, 25-50, and 51-75). Severe head injury was defined as a cranial AIS $\geq 3$. Glasgow Coma Scale (GCS) at admission was also collected and dichotomized (eight or less yes/no). Severe thoracic and abdominal injury were defined as a thoracic or abdominal AIS $\geq 3$, respectively. Mechanism of injury (MOI) was either blunt or penetrating. Hypotension was defined as a Systolic Blood Pressure (SBP) $\leq 89 \mathrm{mmHg}$ at admission to the ED. Data on prehospital care provided by intubation, Physician Helicopter Emergency Medical Services (P-HEMS) assistance, ED intubation and hospital and ICU length of stay (LOS) was also collected. The primary study outcomes were: death within $24 \mathrm{~h}$ after admission and death until hospital discharge. Secondary outcomes were: rate of ICU admission and ICU LOS.

\section{Statistical analysis}

For univariate assessment, independent students $t$ - and chi-squared tests for continuous or categorical variables, respectively, were used on the overall and subgroup population. For skewed variables, median and range were given and a Mann-Whitney-U-test was performed. Backward stepwise logistic regression analyses on the effect of gender on outcome parameters were performed. Odds ratios (ORs) are reported with 95\% confidence intervals $(95 \% \mathrm{CI})$. In addition to gender, the regression models included the covariates age, ISS, year of admission, MOI, prehospital intubation, P-HEMS assistance rate, cranial, thoracic and abdominal AIS $\geq 3$, RTS at the ED, and GCS and SBP on admission. P-values $<0.05$ in two-sided tests were accepted as statistically significant. All analyses were done using IBM SPSS Statistics 23.0. 


\section{Results}

\section{Overall patient characteristics}

A total of 6865 patients were admitted to three Level - 1 trauma centers from 2006 through 2014; 70.6\% was male. Characteristics of the study population are shown in Table 1. Male patients were younger than female patients $(47.7 \pm 20.2$ vs $55.4 \pm 22.0, P<0.0001)$. In the age groups 16 - to 44-years and 45 - to 54-years, males were significantly more represented (Table 2). The median ISS was 25 and $24(P<0.0001)$ for males and females, respectively. Severe head injury was more frequent in females $(71.2 \%$ vs $63.2 \%, P<0.0001)$. An AIS thorax $\geq 3(46.1 \%$ vs $35.1 \%, P<0.0001)$ and an AIS abdomen $\geq 3(11.3 \%$ vs $9.5 \%, P=0.032)$ were more frequent in males. Blunt trauma represented the dominant MOI for both sexes $(92.3 \%$ vs $95.2 \%, P<0.0001)$. Males were more frequently intubated at the scene $(30.9 \%$ vs $27.7 \%$, $P=0.009)$. P-HEMS assistance was significantly more frequent in males $(38.1 \%$ vs $29.0 \%, P<0.0001)$. Males had a higher rate of being intubated whilst in the ED (43.7\% vs $39.3 \%, P=0.003$ ). Furthermore, hypotension at admission to the ED was also more frequent in males (6.8\% vs $4.9 \%, P=0.008)$.

\section{Overall clinical outcomes}

No differences for death until hospital discharge were found in the overall group $(18.5 \%$ vs $17.5 \%$,
$P=0.379)$. No difference in $24-\mathrm{h}$ mortality rate was found between females and males $(6.9 \%$ vs $7.6 \%$, $P=0.334$ ) (Table 3). Females had a significantly lower ICU admission rate $(49.3 \%$ vs $54.5 \%, P<$ 0.0001). The median ICU LOS was two days for males (range 0-184 days) and females (0-200 days), $P<0.0001)$.

The overall association between 24-h mortality and gender Backward logistic regression analysis did not show gender as independently associated with 24-h mortality (OR 1.049; 95\% CI 0.829-1.327; $P=0.693$; Table 4). However, age, P-HEMS assistance, blunt injury, ISS, AIS thorax and abdomen $\geq 3$, hypotension at ED admission ( $\mathrm{SBP} \leq 89 \mathrm{mmHg})$, and prehospital intubation were identified as independent predictors for death. No statistically significant difference was found for gender when we varied the age threshold for defining the subgroups (OR 1.100, 95\% CI 0.867-1.394; $P=0.433$; Table 5).

\section{The overall association between in-hospital mortality and gender}

Backward logistic regression analysis did not show gender as independent risk factor for in-hospital mortality in the overall study population (OR 1.020; 95\% CI

Table 1 Patient characteristics by gender

\begin{tabular}{|c|c|c|c|c|}
\hline & Total $(n=6865)$ & Males $(n=4851)$ & Females $(n=2014)$ & $P$-value \\
\hline Age (years) mean $\pm \mathrm{SD}$ & $49.9 \pm 21.0$ & $47.7 \pm 20.2$ & $55.4 \pm 22.0$ & $<0.0001$ \\
\hline \multicolumn{5}{|l|}{ Age groups (\%) } \\
\hline $16-44$ years & $2861(41.7)$ & $2212(45.5)$ & $652(32.4)$ & $<0.0001$ \\
\hline $45-54$ years & $1083(15.8)$ & $821(16.9)$ & $261(13.0)$ & $<0.0001$ \\
\hline $55+$ years & $2921(42.5)$ & $1818(37.5)$ & $1101(54.7)$ & $<0.0001$ \\
\hline ISS median (range) & $25.0(16-75)$ & $25.0(16-75)$ & $24.0(16-75)$ & $<0.0001$ \\
\hline \multicolumn{5}{|l|}{ ISS categorical (\%) } \\
\hline $16-24$ & $3417(49.8)$ & $2376(49.0)$ & $1041(51.7)$ & 0.044 \\
\hline $25-50$ & $3309(48.2)$ & $2369(48.8)$ & $940(46.7)$ & 0.108 \\
\hline $51-75$ & $139(2.0)$ & $106(2.2)$ & $33(1.6)$ & 0.171 \\
\hline Blunt mechanism of injury (\%) & $6396(93.2)$ & $4478(92.3)$ & $1918(95.2)$ & $<0.0001$ \\
\hline AlS cranial $\geq 3(\%)$ & $4502(65.6)$ & $3068(63.2)$ & $1434(71.2)$ & $<0.0001$ \\
\hline AIS thorax $\geq 3$ (\%) & $2944(42.9)$ & $2237(46.1)$ & $707(35.1)$ & $<0.0001$ \\
\hline AIS abdomen $\geq 3$ (\%) & $738(10.8)$ & $547(11.3)$ & $191(9.5)$ & 0.032 \\
\hline $\mathrm{SBP} \leq 89 \mathrm{mmHg}$ at admission (\%) & $429(6.2)$ & $330(6.8)$ & $99(4.9)$ & 0.008 \\
\hline GCS at admission median (range) & $14.0(3-15)$ & $14.0(3-15)$ & $14.0(3-15)$ & 0.048 \\
\hline P-HEMS assistance (\%) & $2432(35.4)$ & $1847(38.1)$ & $585(29.0)$ & $<0.0001$ \\
\hline Prehospital intubation (\%) & $2057(30.0)$ & $1500(30.9)$ & $557(27.7)$ & 0.009 \\
\hline Intubation at ED (\%) & $2912(42.4)$ & $2120(43.7)$ & $792(39.3)$ & 0.003 \\
\hline
\end{tabular}

AIS, Abbreviated Injury Scale; ED, Emergency Department; GCS, Glasgow Coma Scale; ISS, Injury Severity Score; P-HEMS, Physician staffed Helicopter Emergency Medical Service; SBP, Systolic Blood Pressure; SD, Standard Deviation 
Table 2 Patient characteristics by gender among those aged 16-44-years old

\begin{tabular}{|c|c|c|c|c|}
\hline & Total $(n=2861)$ & Males $(n=2209)$ & Females $(n=652)$ & $P$-value \\
\hline Age (years) mean $\pm S D$ & $29.0 \pm 8.4$ & $29.1 \pm 8.4$ & $28.6 \pm 8.6$ & 0.180 \\
\hline ISS median (range) & $25.0(16-75)$ & $25.0(16-75)$ & $25.0(16-75)$ & 0.072 \\
\hline \multicolumn{5}{|l|}{ ISS categorical (\%) } \\
\hline $16-24$ & $1348(47.1)$ & $1024(46.4)$ & $324(49.7)$ & 0.134 \\
\hline $25-50$ & $1428(49.9)$ & $1118(50.6)$ & $310(47.5)$ & 0.169 \\
\hline $51-75$ & $85(3.0)$ & $67(3.0)$ & $18(2.8)$ & 0.719 \\
\hline Blunt mechanism of injury (\%) & $2580(90.2)$ & $1971(89.2)$ & 609 (93.4) & 0.002 \\
\hline AIS cranial $\geq 3$ (\%) & $1704(59.6)$ & $1313(59.4)$ & $391(60.0)$ & 0.808 \\
\hline AIS thorax $\geq 3$ (\%) & 1455 (50.9) & $1140(51.6)$ & $315(48.3)$ & 0.139 \\
\hline AIS abdomen $\geq 3$ (\%) & $474(16.6)$ & $358(16.2)$ & $116(17.8)$ & 0.339 \\
\hline $\mathrm{SBP} \leq 89 \mathrm{mmHg}$ at admission (\%) & $201(7.0)$ & $165(7.5)$ & $36(5.5)$ & 0.217 \\
\hline GCS at admission median (range) & $13.0(3-15)$ & $13.0(3-15)$ & $14.0(3-15)$ & 0.110 \\
\hline P-HEMS assistance (\%) & $1218(42.6)$ & $963(43.6)$ & $255(39.1)$ & 0.110 \\
\hline Prehospital intubation (\%) & $895(31.3)$ & $709(32.1)$ & $186(28.5)$ & 0.225 \\
\hline Intubation at ED (\%) & $1285(44.9)$ & $1012(45.8)$ & $273(41.9)$ & 0.184 \\
\hline
\end{tabular}

AIS, Abbreviated Injury Scale; ED, Emergency Department; GCS, Glasgow Coma Scale; ISS, Injury Severity Score; P-HEMS, Physician staffed Helicopter Emergency Medical Service; SBP, Systolic Blood Pressure; SD, Standard Deviation

$0.865-1.024 ; P=0.811)$. The same variables that were identified as independent predictors for 24-h mortality, were also independent predictors for in-hospital mortality (Table 4). No statistically significant difference was found for gender when we varied the age threshold for defining the subgroups (OR 1.085; 95\% CI 0.917-1.283; $P=0.341$; Table 5).

\section{The overall association between ICU admission and gender}

Backward logistic regression analysis showed that the male gender was independently associated with a $20.5 \%$ increased likelihood for ICU admission compared to the female gender (OR 1.205; 95\% CI 1.05-1.39; $P=0.010$; Table 4). A statistically significant difference was found for gender when we varied the age threshold for defining the subgroups (OR 1.192; 95\% CI 1.035-1.374; $P=0.015$; Table 5).

\section{Patient characteristics of those aged 16- to 44-years}

Females and males were found to have comparable age, ISS, GCS, and AIS (Table 2). Females had a significantly higher rate of blunt injury $(93.4 \%$ vs $89.2 \%, P=0.002)$. The rates of hypotension, P-HEMS assistance and (prehospital) intubation were similar.

\section{Clinical outcomes in the 16- to 44 age group}

Females did have significantly lower in-hospital mortality rates than to males $(10.4 \%$ vs $13.4 \%, P=0.046)$ (Table 6). No significant differences in 24-h mortality, ICU LOS and admission, or hospital LOS were found between men and women.

\section{The association between $24-\mathrm{h}$ mortality and gender in the $16-$ to 44 age group}

Backward logistic regression analysis did not show gender independently associated with 24-h mortality (OR 0.939; 95\% CI 0.567-1.554, $P=0.806$ ). When we varied the age threshold (i.e. 16-40 age group), no statistically significant differences were found (Table 7). In this group, other variables were identified as independent predictors; blunt injury, ISS, $\mathrm{SBP} \leq 89 \mathrm{mmHg}$, RTS $\leq 12$, and AIS cranial and thorax $\geq 3$ (Table 8.)

Table 3 Clinical outcome comparison by gender

\begin{tabular}{lllll}
\hline & Total $(n=6865)$ & Males $(n=4851)$ & Females $(n=2014)$ & $P$-value \\
\hline LOS (days) mean (range) & $15.0(0-220)$ & $15.3(0-202)$ & $14.2(0-220)$ & 0.070 \\
ICU stay (days) mean (range) & $5.7(0-200)$ & $5.8(0-184)$ & $5.3(0-200)$ & $<0.0001$ \\
ICU admission rate (\%) & $3638(53.0)$ & $2646(54.5)$ & $992(49.3)$ & $<0.001$ \\
In-hospital mortality rate (\%) & $1223(17.8)$ & $851(17.5)$ & $372(18.5)$ & 0.379 \\
24h mortality rate (\%) & $508(7.4)$ & $369(7.6)$ & $139(6.9)$ & 0.334 \\
\hline
\end{tabular}

ICU, Intensive Care Unit; LOS, Length of Stay 
Table 4 Clinical outcome comparison by gender among those aged 16-44-years old

\begin{tabular}{lllll}
\hline & Total $(n=2861)$ & Males $(n=2209)$ & Females $(n=652)$ & $P$-value \\
\hline LOS (days) mean (range) & $15.9(0-220)$ & $15.6(0-201)$ & $17.3(0-220)$ & 0.081 \\
ICU stay (days) mean (range) & $6.1(0-200)$ & $5.8(0-114)$ & $1275(57.7)$ & $374(57.4)$ \\
ICU admission rate (\%) & $1649(57.6)$ & $296(13.4)$ & $68(10.4)$ & 0.651 \\
In-hospital mortality rate (\%) & $364(12.7)$ & $159(7.2)$ & $33(5.1)$ & 0.458 \\
24h mortality rate (\%) & $192(6.7)$ & 0.046 \\
\hline
\end{tabular}

ICU, Intensive Care Unit; LOS, Length of Stay

The association between in-hospital mortality and gender in the 16- to 44 age group

Gender was not independently associated with inhospital mortality in this age group (OR 1.158; 95\% CI 0.827-1.621; $P=0.393$; Table 8 ). When we varied the age threshold (i.e. 16-40 age group), no statistically significant differences were found (Table 7). Blunt injury, ISS, prehospital intubation, hypotension at ED admission (SBP $\leq 89 \mathrm{mmHg}), \quad \mathrm{GCS} \leq 8$, admission year and AIS cranial, thorax and abdomen $\geq 3$ were found independent predictors.

\section{The association between ICU admission and gender in the 16 - to 44 age group}

The male gender was not independently associated with ICU admission rate (OR 0.982; 95\% CI 0.799-1.208; $P=0.867$ ). When we varied the age threshold (i.e. $16-40$ age group), no statistically significant differences were

Table 5 Logistic regression analysis of 24h mortality, in-hospital mortality and ICU admission on gender and other covariates in trauma patients (ISS $\geq 16)$

\begin{tabular}{|c|c|c|c|c|c|c|c|c|c|}
\hline \multirow[b]{2}{*}{ Variable } & \multicolumn{3}{|c|}{ 24h mortality } & \multicolumn{3}{|c|}{ In-hospital mortality } & \multicolumn{3}{|c|}{ ICU admission } \\
\hline & $\overline{\mathrm{OR}}$ & $95 \% \mathrm{Cl}$ & $P$ - value & $\overline{\mathrm{OR}}$ & $95 \% \mathrm{Cl}$ & $P$ - value & $\overline{\mathrm{OR}}$ & $95 \% \mathrm{Cl}$ & $P$ - value \\
\hline Male Gender & 1.049 & $0.829-1.327$ & 0.693 & 1.020 & $0.865-1.204$ & 0.811 & 1.205 & $1.046-1.388$ & 0.010 \\
\hline \multicolumn{10}{|l|}{ Age, years } \\
\hline $16-44$ & REF & & $<0.0001^{a}$ & REF & & $<0.0001^{\mathrm{a}}$ & REF & & $0.002^{\mathrm{a}}$ \\
\hline $45-54$ & 0.972 & $0.688-1.373$ & 0.872 & 1.232 & $0.961-1.580$ & 0.100 & 0.906 & $0.749-1.096$ & 0.311 \\
\hline $55+$ & 2.179 & $1.715-2.767$ & $<0.0001$ & 3.922 & $3.284-4.684$ & $<0.0001$ & 0.770 & $0.665-0.891$ & $<0.0001$ \\
\hline Blunt MOI & 0.477 & $0.338-0.674$ & $<0.0001$ & 0.561 & $0.422-0.745$ & $<0.0001$ & 0.734 & $0.562-0.959$ & 0.023 \\
\hline P-HEMS & 1.321 & $1.042-1.675$ & 0.021 & 1.212 & $1.021-1.439$ & 0.028 & 1.234 & $1.057-1.441$ & 0.008 \\
\hline \multicolumn{10}{|l|}{ Admission at ED, year } \\
\hline 2004-2006 & 1.548 & $0.800-2.992$ & 0.194 & 1.315 & $0.826-2.094$ & 0.249 & 0.724 & $0.492-1.066$ & 0.102 \\
\hline 2007-2009 & 2.214 & $1.615-3.035$ & $<0.0001$ & 1.533 & & $<0.0001$ & 1.014 & $0.832-1.235$ & 0.893 \\
\hline 2010-2012 & 1.441 & $1.070-1.940$ & 0.016 & 1.224 & $1.230-1.909$ & 0.049 & 0.615 & $0.523-0.724$ & $<0.0001$ \\
\hline 2013-2014 & REF & & $<0.0001^{\mathrm{a}}$ & REF & $1.001-1.498$ & $<0.0001^{\mathrm{a}}$ & REF & & $<0.0001^{\mathrm{a}}$ \\
\hline \multicolumn{10}{|l|}{ ISS } \\
\hline $16-24$ & REF & & $<0.0001^{\mathrm{a}}$ & REF & & $<0.0001^{\mathrm{a}}$ & REF & & $<0.0001^{a}$ \\
\hline $25-50$ & 2.985 & $2.235-3.987$ & $<0.0001$ & 3.653 & $3.029-4.407$ & $<0.0001$ & 2.190 & $1.898-2.526$ & $<0.0001$ \\
\hline $51-75$ & 3.873 & $2.302-6.514$ & $<0.0001$ & 8.657 & $5.646-13.274$ & $<0.0001$ & 2.126 & $1.139-3.970$ & 0.018 \\
\hline AIS Thorax $\geq 3$ & 0.647 & $0.514-0.814$ & $<0.0001$ & 0.593 & 0.499-0.705 & $<0.0001$ & 2.053 & $1.757-2.399$ & $<0.0001$ \\
\hline AIS Abdomen $\geq 3$ & 1.646 & $1.202-2.255$ & 0.002 & 1.405 & $1.088-1.813$ & 0.009 & 1.776 & $1.406-2.245$ & $<0.0001$ \\
\hline Prehospital intubation & 2.042 & $1.605-2.598$ & $<0.0001$ & 1.674 & $1.405-1.994$ & $<0.0001$ & 0.756 & $0.640-0.893$ & 0.001 \\
\hline $\mathrm{SBP} \leq 89 \mathrm{mmHg}$ at ED & 5.502 & $4.179-7.245$ & $<0.0001$ & 3.030 & $2.358-3.895$ & $<0.0001$ & 0.372 & $0.281-0.494$ & $<0.0001$ \\
\hline $\mathrm{GCS} \leq 8$ at ED & 9.221 & $6.287-13.526$ & $<0.0001$ & 5.200 & 4.139-6.532 & $<0.0001$ & 2.262 & $1.841-2.778$ & $<0.0001$ \\
\hline RTS $\leq 12$ at ED & 1.034 & $0.667-2.670$ & 0.880 & 1.352 & $1.034-1.767$ & 0.027 & 3.460 & $2.844-4.209$ & $<0.0001$ \\
\hline AIS Head $\geq 3$ & & & & 1.479 & $1.196-1.829$ & $<0.0001$ & 1.155 & $0.977-1.365$ & 0.091 \\
\hline
\end{tabular}

${ }^{a} P$ - value represents the overall significance across the categories

AIS, Abbreviated Injury Scale; CI, Confidence Interval; ED, Emergency Department; GCS, Glasgow Coma Scale; ISS, Injury Severity Score; MOI, Mechanism of Injury; P-HEMS, Physician staffed Helicopter Emergency Services; OR, Odds ratio; SBP, Systolic Blood Pressure; RTS, Revised Trauma Score 
Table 6 Logistic regression analysis of 24h mortality, in-hospital mortality and ICU admission on gender and other covariates in trauma patients (ISS $\geq 16$ ) aged 16-44-years

\begin{tabular}{|c|c|c|c|c|c|c|c|c|c|}
\hline \multirow[b]{2}{*}{ Variable } & \multicolumn{3}{|c|}{ 24h mortality } & \multicolumn{3}{|c|}{ In-hospital mortality } & \multicolumn{3}{|c|}{ ICU admission } \\
\hline & $\mathrm{OR}$ & $95 \% \mathrm{Cl}$ & $P$-value & $\mathrm{OR}$ & $95 \% \mathrm{Cl}$ & $P$-value & $\overline{\mathrm{OR}}$ & $95 \% \mathrm{Cl}$ & $P$-value \\
\hline Male Gender & 0.939 & $0.567-1.554$ & 0.806 & 1.158 & $0.827-1.621$ & 0.393 & 0.982 & $0.799-1.208$ & 0.867 \\
\hline Blunt $\mathrm{MOI}$ & 0.231 & $0.107-0.498$ & $<0.0001$ & 0.327 & $0.215-0.497$ & $<0.0001$ & 1.563 & $0.927-2.638$ & 0.094 \\
\hline \multicolumn{10}{|l|}{ ISS } \\
\hline $16-24$ & REF & & $<0.0001^{\mathrm{a}}$ & REF & & $<0.0001^{\mathrm{a}}$ & REF & & $<0.0001^{a}$ \\
\hline $25-50$ & 6.546 & $3.447-12.432$ & $<0.0001$ & 3.769 & $2.527-5.621$ & $<0.0001$ & 3.463 & $2.769-4.332$ & $<0.0001$ \\
\hline $51-75$ & 8.090 & 2.153-30.399 & 0.002 & 7.858 & $4.105-15.041$ & $<0.0001$ & 9.550 & $4.370-20.868$ & $<0.0001$ \\
\hline Prehospital intubation & & & & 2.305 & $1.687-3.149$ & $<0.0001$ & 1.262 & $1.000-1.593$ & 0.050 \\
\hline $\mathrm{SBP} \leq 89 \mathrm{mmHg}$ at $\mathrm{ED}$ & 4.173 & $2.186-7.965$ & $<0.0001$ & 4.638 & $3.096-6.949$ & $<0.0001$ & 1.807 & $1.233-2.647$ & 0.002 \\
\hline $\mathrm{GCS} \leq 8$ at $\mathrm{ED}$ & 4.251 & $2.200-8.215$ & $<0.0001$ & 13.266 & $7.688-22.890$ & $<0.0001$ & 4.434 & $3.365-5.844$ & $<0.0001$ \\
\hline RTS $\leq 12$ at ED & 3.293 & $1.340-8.094$ & 0.009 & 0.868 & $0.476-1.585$ & 0.646 & 1.615 & $1.167-2.234$ & 0.004 \\
\hline AlS Head $\geq 3$ & 1.795 & $0.978-3.296$ & 0.059 & 2.189 & $1.491-3.215$ & $<0.0001$ & & & \\
\hline AIS Thorax $\geq 3$ & 0.603 & $0.363-1.004$ & 0.052 & 0.601 & $0.446-0.812$ & 0.001 & 0.569 & $0.453-0.714$ & $<0.0001$ \\
\hline AlS Abdomen $\geq 3$ & & & & 1.565 & $1.076-2.276$ & 0.019 & 1.532 & $1.006-2.333$ & 0.047 \\
\hline \multicolumn{10}{|l|}{ Admission at ED, year } \\
\hline $2004-2006$ & & & & 1.247 & $0.524-2.968$ & 0.617 & & & \\
\hline $2007-2009$ & & & & 2.489 & $1.654-3.746$ & $<0.0001$ & & & \\
\hline 2010-2012 & & & & 1.775 & $1.209-2.605$ & 0.003 & & & \\
\hline 2013-2014 & & & & REF & & $<0.0001^{\mathrm{a}}$ & & & \\
\hline
\end{tabular}

${ }^{a} P$ - value represents the overall significance across the categories

AIS, Abbreviated Injury Scale; CI, Confidence Interval; ED, Emergency Department; GCS, Glasgow Coma Scale; ISS, Injury Severity Score; MOI, Mechanism of Injury; OR, Odds ratio; SBP, Systolic Blood Pressure; RTS, Revised Trauma Score

found (Table 7). ISS, prehospital intubation, hypotension at ED admission ( $\mathrm{SBP} \leq 89 \mathrm{mmHg}), \mathrm{GCS} \leq 8, \mathrm{RTS} \leq 12$, and AIS cranial, thorax and abdomen $\geq 3$ were identified as independent predictors (Table 8).

\section{Discussion}

There is an increasing amount of laboratory and clinical data, suggesting gender dimorphism in severely injured patients $[1-7,9]$.

This study is the first multicenter analysis performed on this subject in the Netherlands. The advantage of this retrospective study is the large sample size over an extended study period.

In this study, a statistically significant survival advantage was found in female patients age 16 to 44-years old. Overall, females had significantly lower ICU admission rates. Logistic regression analyses did not show gender as an independent predictor for early and in-hospital mortality in any subset population. However, the male gender was independently associated with a $20.5 \%$ increased likelihood for ICU admission. In this study, patients were divided in three age groups; 16 - to 44years old, 45- to 54- years old, and 55- years old or older. These categories were surrogates for the pre-, peri- and postmenopausal stages and were defined based on literature findings $[19,23]$. As stated before, we varied the age thresholds for defining these groups. We found that varying these thresholds did not changed our results, therefore we continued using the previous stated groups (16- to 44- years old, 45- to 54- years old, and 55 - years old or older). Women one year after the onset of menopause show a decline in estrogen levels, which may explain the absence of beneficial effects in women 55 -years old or older in our population [1, 3, 15]. Considering postmenopausal women using hormone replacement therapy (HRT) a minority, it seems unlikely that this would have affected the results of the current study. To investigate the influence of aging, we excluded every patient who was 19 years or younger, or 61 or older in a subgroup analysis. The population that remained included 4132 patients. In-hospital mortality, mortality within $24 \mathrm{~h}$ and ICU admission, did not show a statistically significant difference. As described earlier, some studies found that severely injured men of 50- years or younger had a greater chance of not surviving (27\%) than women of the same age [11]. This survival advantage for women of 50 years or younger was attributed to interactions between the immune and endocrine systems of both sexes, and not to a difference in MOI, severity or pattern [11]. 
Table 7 Logistic regression analysis of $<24 \mathrm{~h}$ mortality, in-hospital mortality and ICU admission on gender and other covariates in trauma patients (ISS $\geq 16)$

\begin{tabular}{|c|c|c|c|c|c|c|c|c|c|}
\hline \multirow[b]{2}{*}{ Variable } & \multicolumn{3}{|c|}{$<24 \mathrm{~h}$ mortality } & \multicolumn{3}{|c|}{ In-hospital mortality } & \multicolumn{3}{|c|}{ ICU admission } \\
\hline & $\mathrm{OR}$ & $95 \% \mathrm{Cl}$ & $P$ - value & $\mathrm{OR}$ & $95 \% \mathrm{Cl}$ & $P$ - value & $\overline{O R}$ & $95 \% \mathrm{Cl}$ & $P$ - value \\
\hline Male Gender & 1.100 & $0.867-1.394$ & 0.433 & 1.085 & $0.917-1.283$ & 0.341 & 1.192 & $1.035-1.374$ & 0.015 \\
\hline \multicolumn{10}{|l|}{ Age, years } \\
\hline $16-40$ & REF & REF & $<0.0001^{\mathrm{a}}$ & REF & REF & $<0.0001^{\mathrm{a}}$ & REF & REF & $<0.0001^{\mathrm{a}}$ \\
\hline $41-60$ & 1.147 & $0.866-1.518$ & 0.338 & 1.468 & $1.196-1.802$ & $<0.0001$ & 0.876 & $0.746-1.029$ & 0.108 \\
\hline $60+$ & 2.784 & $2.142-3.619$ & $<0.0001$ & 5.336 & 4.381-6.499 & $<0.0001$ & 0.708 & $0.603-0.832$ & $<0.0001$ \\
\hline Blunt MOI & 0.462 & $0.327-0.654$ & $<0.0001$ & 0.539 & $0.405-0.717$ & $<0.0001$ & 0.739 & $0.566-0.965$ & 0.027 \\
\hline P-HEMS & 1.321 & $1.041-1.676$ & 0.022 & 1.217 & $1.024-1.447$ & 0.026 & 1.227 & $1.051-1.433$ & 0.010 \\
\hline \multicolumn{10}{|l|}{ Admission at ED, year } \\
\hline $2004-2006$ & 1.593 & $0.820-3.095$ & 0.170 & 1.382 & $0.864-2.211$ & 0.177 & 0.720 & $0.489-1.061$ & 0.097 \\
\hline 2007-2009 & 2.269 & $1.651-3.119$ & $<0.0001$ & 1.589 & $1.272-1.984$ & $<0.0001$ & 1.011 & $0.830-1.232$ & 0.914 \\
\hline 2010-2012 & 1.463 & $1.085-1.974$ & 0.013 & 1.244 & $1.014-1.526$ & 0.036 & 0.615 & $0.523-0.723$ & $<0.0001$ \\
\hline 2013-2014 & REF & REF & $<0.0001^{\mathrm{a}}$ & REF & REF & $<0.0001$ & REF & REF & $<0.0001$ \\
\hline \multicolumn{10}{|l|}{ ISS } \\
\hline $16-24$ & REF & REF & $<0.0001^{\mathrm{a}}$ & REF & REF & $<0.0001$ & REF & REF & $<0.0001^{\mathrm{a}}$ \\
\hline $25-50$ & 3.069 & $2.288-4.117$ & $<0.0001$ & 3.692 & $3.055-4.463$ & $<0.0001$ & 2.199 & $1.906-2.538$ & $<0.0001$ \\
\hline $51-75$ & 4.083 & $2.414-6.905$ & $<0.0001$ & 8.834 & $5.745-13.585$ & $<0.0001$ & 2.109 & $1.130-3.938$ & 0.019 \\
\hline AIS Thorax $\geq 3$ & 0.645 & $0.509-0.819$ & $<0.0001$ & 0.608 & $0.510-0.724$ & $<0.0001$ & 2.048 & $1.752-2.394$ & $<0.0001$ \\
\hline AIS Abdomen $\geq 3$ & 1.667 & $1.207-2.304$ & 0.002 & 1.492 & $1.154-1.929$ & 0.002 & 1.756 & $1.389-2.220$ & $<0.0001$ \\
\hline Prehospital intubation & 2.095 & $1.645-2.669$ & $<0.0001$ & 1.721 & $1.443-2.053$ & $<0.0001$ & 0.755 & $0.639-0.892$ & 0.001 \\
\hline $\mathrm{SBP} \leq 89 \mathrm{mmHg}$ at ED & 5.456 & $4.140-7.188$ & $<0.0001$ & 2.976 & $2.315-3.827$ & $<0.0001$ & 0.374 & $0.282-0.496$ & $<0.0001$ \\
\hline $\mathrm{GCS} \leq 8$ at ED & 9.535 & 6.496-13.998 & $<0.0001$ & 5.464 & $4.335-6.886$ & $<0.0001$ & 2.260 & $1.840-2.776$ & $<0.0001$ \\
\hline $\mathrm{RTS} \leq 12$ at ED & 1.029 & $0.663-1.597$ & 0.897 & 1.357 & $1.036-1.777$ & 0.027 & 3.464 & $2.848-4.215$ & $<0.0001$ \\
\hline AIS Head $\geq 3$ & & & & 1.461 & $1.180-1.810$ & 0.001 & 1.163 & $0.984-1.374$ & 0.077 \\
\hline
\end{tabular}

AIS, Abbreviated Injury Scale; Cl, Confidence Interval; ED, Emergency Department; GCS, Glasgow Coma Scale; ISS, Injury Severity Score; MOI, Mechanism of Injury; P-HEMS, Physician staffed Helicopter Emergency Services; OR, Odds ratio; SBP, Systolic Blood Pressure; RTS, Revised Trauma Score

a $P$ - value represents the overall significance across the categories

Similarly, a meta-analysis of 19 studies found a survival advantage for women older than 50 years with a low to moderate injury (ISS $\leq 25$ ). Interestingly, this advantage was not shown in severe trauma [33]. In a large retrospective cohort study it was demonstrated that female blunt trauma patients with an ISS $\geq 16$ and relevant bleeding had lower rates of multiple organ failure (MOF) and sepsis [6]. After stratification by age group, these findings were most evident in groups of females at reproductive age (i.e., 16-44 years) [6]. They found no differences in the age group 55- to 64-years old. However, the possibility of late onset menopause or HRT in these women cannot be ruled out completely [6].

Another study stratified severely injured patients by MOI and age, assuming women of 50 -years or older to be postmenopausal [8]. A major difference compared to the current study is that they excluded patients ages 41to 50 years old because women in this age group were most likely to be in the perimenopausal stage of their menstrual cycle. The authors found a survival advantage for women aged below 40 years old and with an ISS between 16 and 24, although not statistically significant [8]. Logistic regression analyses also failed to identify gender as independently associated with mortality and are therefore consistent with our results.

Significant differences in mechanism and pattern of injury among male and female patients were found in our population. Females had more AIS $\geq 3$ injuries to the head (71.2\%) and males had more AIS $\geq 3$ injuries to the chest (46.1\%) and abdomen (11.3\%). Blunt trauma represented the dominant mechanism of injury for both male and female patients $(92.3 \%$ vs $95.2 \%, P<0.0001)$. Male patients suffered more often from motorcycle accidents (7.3\% vs $3.3 \%)$ and penetrating injuries $(5.2 \%$ vs $2.8 \%)$. Penetrating trauma may differ from blunt by treatment and associated injuries. Since a small group suffered from penetrating injuries, the data reported would be unlikely to reveal a significant difference if these were analysed solely. Our study therefore did 
Table 8 Logistic regression analysis of <24h mortality, in-hospital mortality and ICU admission on gender and other covariates in trauma patients (ISS $\geq 16$ ) aged 16- to 40-years

\begin{tabular}{|c|c|c|c|c|c|c|c|c|c|}
\hline \multirow[b]{2}{*}{ Variable } & \multicolumn{3}{|c|}{ Early $(<24 \mathrm{~h})$ mortality } & \multicolumn{3}{|c|}{ In-hospital mortality } & \multicolumn{3}{|c|}{ ICU admission } \\
\hline & $\overline{\mathrm{OR}}$ & $95 \% \mathrm{Cl}$ & $P$-value & $\overline{O R}$ & $95 \% \mathrm{Cl}$ & $P$-value & $\overline{O R}$ & $95 \% \mathrm{Cl}$ & $P$-value \\
\hline Male Gender & 1.498 & $0.903-2.484$ & 0.117 & 1.224 & $0.847-1.767$ & 0.282 & 1.057 & $0.810-1.379$ & 0.682 \\
\hline Blunt MOI & 2.377 & $1.425-3.964$ & 0.001 & 3.196 & $2.055-4.968$ & $<0.0001$ & & & \\
\hline \multicolumn{10}{|l|}{ ISS } \\
\hline $16-24$ & REF & REF & 0.009 & REF & REF & $<0.0001^{a}$ & REF & REF & $<0.0001^{a}$ \\
\hline $25-50$ & 2.234 & $1.314-3.798$ & 0.003 & 3.408 & $2.217-5.239$ & $<0.0001$ & 2.256 & $1.754-2.902$ & $<0.0001$ \\
\hline $51-75$ & 2.610 & $1.168-5.834$ & 0.019 & 6.834 & $3.404-13.722$ & $<0.0001$ & 1.432 & $0.628-3.265$ & 0.393 \\
\hline Prehospital intubation & 3.304 & $2.109-5.176$ & $<0.0001$ & 2.376 & $1.700-3.322$ & $<0.0001$ & 0.576 & $0.430-0.773$ & $<0.0001$ \\
\hline $\mathrm{SBP} \leq 89 \mathrm{mmHg}$ at ED & 7.482 & $4.645-12.052$ & $<0.0001$ & 4.278 & $2.780-6.583$ & $<0.0001$ & 0.302 & $0.188-0.485$ & $<0.0001$ \\
\hline $\mathrm{GCS} \leq 8$ at $\mathrm{ED}$ & 15.046 & $7.050-32.111$ & $<0.0001$ & 14.074 & $7.740-25.593$ & $<0.0001$ & 2.828 & $1.969-4.063$ & $<0.0001$ \\
\hline RTS $\leq 12$ at ED & & & & 0.842 & $0.436-1.625$ & 0.608 & 4.390 & $3.089-6.238$ & $<0.0001$ \\
\hline AlS Head $\geq 3$ & & & & 0.495 & $0.328-0.747$ & 0.001 & & & \\
\hline AIS Thorax $\geq 3$ & 1.676 & $1.107-2.537$ & 0.015 & 1.520 & $1.100-2.101$ & 0.011 & 0.532 & $0.418-0.678$ & $<0.0001$ \\
\hline AIS Abdomen $\geq 3$ & 0.589 & $0.365-0.949$ & 0.030 & 0.593 & $0.399-0.881$ & 0.010 & 0.527 & $0.382-0.729$ & $<0.0001$ \\
\hline \multicolumn{10}{|l|}{ Admission at ED, year } \\
\hline $2004-2006$ & 0.768 & $0.190-3.098$ & 0.711 & 1.101 & $0.425-2.852$ & 0.843 & 0.711 & $0.371-1.362$ & 0.304 \\
\hline $2007-2009$ & 3.543 & $1.957-6.414$ & $<0.0001$ & 2.790 & $1.788-4.355$ & $<0.0001$ & 0.863 & $0.606-1.230$ & 0.415 \\
\hline 2010-2012 & 1.522 & $0.848-2.732$ & 0.160 & 1.749 & $1.145-2.674$ & 0.010 & 0.473 & $0.352-0.635$ & $<0.0001$ \\
\hline 2013-2014 & REF & REF & $<0.0001^{\mathrm{a}}$ & REF & REF & $<0.0001^{\mathrm{a}}$ & REF & REF & $<0.0001^{\mathrm{a}}$ \\
\hline
\end{tabular}

AIS, Abbreviated Injury Scale; CI, Confidence Interval; ED, Emergency Department; GCS, Glasgow Coma Scale; ISS, Injury Severity Score; MOI, Mechanism of Injury; OR, Odds ratio; SBP, Systolic Blood Pressure; RTS, Revised Trauma Score

${ }^{a} P$ - value represents the overall significance across the categories

not further investigate the different trauma mechanisms (for instance whether it was a fall from height or a motor cycle accident) because it was not the main purpose of this article.

The current study also found that the male gender was an independent predictor of ICU admission after correction for several clinical important confounders. A probable explanation could be that some unknown confounders, such as hormonal status, were not included in the analysis. However, studies demonstrated that younger males (aged 45- years or younger) showed a significant higher frequency of MOF and mortality and also showed a longer ICU stay than females [24, 31]. An earlier study showed a significantly higher ICU admission rate for male surgical intensive care patients $(64.2 \%$ vs $35.8 \%)$. Therefore they state that despite comparable age and surgical procedures, female patients may not be as critically ill as male patients $[35,36]$. One clinical prospective cohort managed to measure sex steroids during ICU stay of severely injured patients and assess mortality [34]. They found a significantly elevated estradiol level in non-survivors, with the most severe injured patients having the highest levels [34]. Furthermore, after comparison with other parameters, the ability of estradiol to predict death was comparable with the ISS.
One limitation of their study is that only a single estradiol level was drawn, $48 \mathrm{~h}$ after admission [34]. It is however, not known how quickly estrogens are elevated [34].

However, we also believe that whether or not the trauma patient is admitted to the ICU is influenced mostly by the physician on duty and cannot be solely be attributed to medical status such as injury severity or trauma mechanism. We therefore can only speculate that hormonal status may be considered a risk factor for ICU admission in severely injured patients.

Previous clinical studies, investigating gender-based differences in severely injured patients were often unable to produce results resembling the experimental data $[2,6-8,10-12,14-16,30-32]$.

Experimental studies in rodents have demonstrated the female gender to be protective after severe injury and hemorrhage [1-3]. Other studies demonstrated that resistance to trauma or hemorrhage induced organ injury, varies during the menstrual cycle, with maximal protection during the proestrus stage, when estrogen levels are the highest $[1,3,5]$. However, when estrogen exceeded the physiological level in female rodents after trauma-hemorrhage, resembling pregnancy and burn injuries, the immune response was down-regulated [7-9, 20, 21]. 
These findings suggest that high end physiological levels of estrogen are considered beneficial on survival after trauma or hemorrhage [2, 3, 22, 29].

The retrospective nature of this study has some limitations. Since the retrospective trauma registry did not collect any data on reproductive cycle or hormonal status, we divided the patients in three subgroups, as alternates for hormonal status. Women younger than 55- years old were assumed to be pre- or perimenopausal and those aged 55- years or older to be postmenopausal. Another limitation is the lack of information on comorbidity and medication. Factors such as obesity, surgical and medical history, smoking habits, and the use of contraceptive or HRT are known to influence the actual hormonal status and could be possible confounders $[8,10,23,24]$.

We did not stratify the population based on the anatomical sites of injury (and their combinations), since this might lead to so many subgroups that sensitive analysis will not be possible. It is well known that trauma research is hampered by a heterogeneous population. We therefore decided to enter ISS and other surrogates for injury severity (i.e. AIS Cranial, Thorax and Abdomen) in our analysis to cope with the heterogeneity of the trauma patient population.

We are aware that there exist much larger registry studies with over 20,000 cases [5, 6, 8, 11, 14, 28]. Our study consist of 6865 cases. Our sample may be too small to detect a survival advantage for female trauma patients.

We attempted to use as much standardized definitions as possible to create a homogeneous study population with minimal variations. The Shock Index (SI), defined by the ratio of heart rate to systolic blood pressure, is the most commonly used clinical parameter to signal hemorrhagic shock [25-27]. However, the trauma registry lacked data on heart rate at admission for most patients. Therefore, shock was defined as a SBP $\leq 89 \mathrm{mmHg}$ at admission, based on recent literature findings $[8,12,16,24,26]$. Using this definition may have underestimated the effect of hemorrhagic shock on mortality.

We conclude that in this population of severely injured patients, the female gender shows a lower mortality rate in patients aged 16- to 44- years. However, the male gender was independently associated with an increased likelihood for ICU admission. Gender was not independently associated with mortality, despite the large amount of experimental evidence.

Future research should focus on large, prospective multicentre observational studies, in which the actual hormonal status is adequately measured upon admission to the ED, hereby allowing to group patients more precisely by their cycle status and thus observe a clearer hormone-related relationship to their outcome.

\section{Acknowledgements}

Not applicable.

Funding

The authors declare that they did not have any source of funding.

Availability of data and materials

The datasets used and analysed during the current study are available from the corresponding author on reasonable request.

\section{Authors' contributions}

MP collected, analysed and interpreted all the patients data and wrote the article. GG interpreted and helped analysing the patients data and was a major contributor in writing the manuscript. LG designed the study and led the multicenter project, interpreted the patients data and was a major contributor in writing the manuscript. WZ corrected the manuscript and helped with the process of ethical approval at the Medical Research Ethics Committees of the hospital. FB corrected the manuscript. ESML helped MP in analysing the data and corrected the manuscript. EJT, TT and EMML provided the data from the three trauma centers and helped MP in the process of analysing the data. MJRE and MHJV corrected the manuscript and gave their approval for using the data. All authors read and approved the final manuscript.

Ethics approval and consent to participate

This study was approved by the independent Medical Research Ethics Committees of all three hospitals.

Consent for publication

Not applicable

Competing interests

The authors declare that they have no competing interests.

\section{Publisher's Note}

Springer Nature remains neutral with regard to jurisdictional claims in published maps and institutional affiliations.

\section{Author details}

${ }^{1}$ Department of Trauma Surgery, VU University Medical Center, De Boelelaan 1117, P.O. Box 7057, 1007, MB, Amsterdam, The Netherlands. ²Department of Epidemiology and Biostatistics, VU University Medical Center, Amsterdam,

The Netherlands. ${ }^{3}$ Trauma Network North-West, VU University Medical Center, Amsterdam, The Netherlands. ${ }^{4}$ Department of Trauma Surgery, Radboud University Medical Center, Nijmegen, The Netherlands.

${ }^{5}$ Department of Surgery, Erasmus MC, University Medical Center, Rotterdam, The Netherlands. ${ }^{6}$ Trauma Research Unit, Department of Surgery, Erasmus MC, University Medical Center, Rotterdam, The Netherlands.

Received: 3 July 2018 Accepted: 14 January 2019

Published online: 13 February 2019

\section{References}

1. Choudhry M, Bland K, Chaudry I. Trauma and immune response-effect of gender differences. Injury. 2007;38(12):1382-91.

2. Sperry J, Nathens A, Frankel H, Vanek S, Moore E, Maier R, et al. Characterization of the gender dimorphism after injury and hemorrhagic shock: are hormonal differences responsible?*. Crit Care Med. 2008;36(6):1838-45.

3. Angele M, Frantz M, Chaudry I. Gender and sex hormones influence the response to trauma and sepsis: potential therapeutic approaches. Clinics. 2006;61(5).

4. Knöferl M, Angele M, Diodato M, Schwacha M, Ayala A, Cioffi W, et al. Female sex hormones regulate macrophage function after traumahemorrhage and prevent increased death rate from subsequent Sepsis. Ann Surg. 2002;235(1):105-12.

5. Magnotti L, Fischer P, Zarzaur B, Fabian T, Croce M. Impact of gender on outcomes after blunt injury: a definitive analysis of more than 36,000 trauma patients. J Am Coll Surg. 2008;206(5):984-91. 
6. Trentzsch H, Nienaber U, Behnke M, Lefering R, Piltz S. Female sex protects from organ failure and sepsis after major trauma haemorrhage. Injury. 2014;45:S20-8.

7. Yang K, Zhou M, Sperry J, Rong L, Zhu X, Geng L, et al. Significant sex-based outcome differences in severely injured Chinese trauma patients. Shock. 2014:42(1):11-5.

8. Croce M, Fabian T, Malhotra A, Bee T, Miller P. Does gender difference influence outcome? J Trauma. 2002;53(5):889-94.

9. Bird M, Karavitis J, Kovacs E. Sex differences and estrogen modulation of the cellular immune response after injury. Cell Immunol. 2008;252(1-2):57-67.

10. George R, McGwin G, Windham S, Melton S, Metzger J, Chaudry I, et al. Age-related gender differential in outcome after blunt or penetrating trauma. Shock. 2003;19(1):28-32.

11. Wohltmann C, Franklin G, Boaz P, Luchette F, Kearney P, Richardson J, et al. A multicenter evaluation of whether gender dimorphism affects survival after trauma. Am J Surg. 2001;181(4):297-300.

12. Rappold J, Coimbra R, Hoyt D, Potenza B, Fortlage D, Holbrook T. Female gender does not protect blunt trauma patients from complications and mortality. J Trauma. 2002;53(3):436-41.

13. Napolitano L, Greco M, Rodriguez A, Kufera J, West R, Scalea A. Gender differences in adverse outcomes after blunt trauma. J Trauma. 2001;50(2):274-80

14. Trentzsch $\mathrm{H}$, Lefering R, Nienaber U, Kraft R, Faist E, Piltz $\mathrm{S}$. The role of biological sex in severely traumatized patients on outcomes. Ann Surg. 2015;261(4):774-80.

15. Coimbra R, Hoyt D, Potenza B, Fortlage D, Hollingsworth-Fridlund P. Does sexual dimorphism influence outcome of traumatic brain injury patients? The answer is no! J Trauma. 2003;54(4):689-700.

16. George R, McGwin G, Metzger J, Chaudry I, Rue L. The association between gender and mortality among trauma patients as modified by age. J Trauma. 2003;54(3):464-71.

17. Baker S, O'neill B, Haddon W, Long W. The injury severity score. J Trauma. 1974;14(3):187-96.

18. Champion H, Sacco W, Copes W, Gann D, Gennarelli T, Flanagan M. A revision of the trauma score. J Trauma. 1989;29(5):623-9.

19. Weniger M, Angele M, Chaudry I. The role and use of estrogens following trauma. Shock. 2016;46:4-11.

20. May A, Dossett L, Norris P, Hansen E, Dorsett R, Popovsky K, et al. Estradiol is associated with mortality in critically ill trauma and surgical patients. Crit Care Med. 2008:36(1):62-8.

21. Summers J, Ziembicki J, Corcos A, Peitzman A, Billiar T, Sperry J. Characterization of sex dimorphism following severe thermal injury. J Burn Care Res. 2014;35(6):484-90.

22. Sperry J, Minei J. Gender dimorphism following injury: making the connection from bench to bedside. J Leukoc Biol. 2007;83(3):499-506.

23. Nelson H. Menopause. Lancet. 2008;371(9614):760-70.

24. Mostafa G, Huynh T, Sing R, Miles W, Norton H, Thomason M. Genderrelated outcomes in trauma. J Trauma. 2002;53(3):430-5.

25. Mutschler M, Nienaber U, Münzberg M, Wölfl C, Schoechl H, Paffrath T, et al. The shock index revisited - a fast guide to transfusion requirement? A retrospective analysis on 21,853 patients derived from the TraumaRegister DGU®. Crit Care. 2013;17(4):R172.

26. Mutschler M, Nienaber U, Brockamp T, Wafaisade A, Fabian T, Paffrath T, et al. Renaissance of base deficit for the initial assessment of trauma patients: a base deficit-based classification for hypovolemic shock developed on data from 16,305 patients derived from the TraumaRegister DGU๑. Crit Care. 2013;17(2):R42.

27. Rainer T, Li Y, Chan C, Agarwal N, Sin K, Chan S, et al. Validating a pragmatic definition of shock in adult patients presenting to the emergency department. Resuscitation. 2014;85:S113.

28. Haider A, Crompton J, Chang D, Efron D, Haut E, Handly N, et al. Evidence of hormonal basis for improved survival among females with traumaassociated shock: an analysis of the National Trauma Data Bank. J Trauma. 2010;69(3):537-40.

29. Catania R, Angele M, Ayala A, Cioffi W, Bland K, Chaudry I. Dehydroepiandrosterone restores immune function following traumahaemorrhage by a direct effect on T-lymphocytes. Cytokine. 1999; 11(6):443-50

30. Jason Bowles B, Roth B, Demetriades D. Sexual dimorphism in trauma? A retrospective evaluation of outcome. Injury. 2003;34(1):27-31.
31. Frink M, Pape H, van Griensven M, Krettek C, Chaudry I, Hildebrand F. Influence of sex and age on MODS and cytokines after multiple injuries. Shock. 2007;27(2):151-6.

32. Oberholzer A, Keel M, Zellweger R, Steckholzer U, Trentz O, Ertel W. Incidence of septic complications and multiple organ failure in severely injured patients is sex specific. J Trauma. 2000;48(5):932-7.

33. Liu T, Xie J, Yang F, Chen J, Li Z, Yi C, et al. The influence of sex on outcomes in trauma patients: a meta-analysis. Am J Surg. 2015;210(5):911-21.

34. Dossett L, Swenson B, Heffernan D, Bonatti H, Metzger R, Sawyer R, et al. High levels of endogenous estrogens are associated with death in the critically injured adult. J Trauma. 2008;64(3):580-5.

35. Wichmann $M$, Inthorn D, Andress H, Schildberg F. Incidence and mortality of severe sepsis in surgical intensive care patients: the influence of patient gender on disease process and outcome. Intensive Care Med. 2000;26(2):167-72.

36. Brattström O, Granath F, Rossi P, Oldner A. Early predictors of morbidity and mortality in trauma patients treated in the intensive care unit. Acta Anaesthesiol Scand. 2010;54(8):1007-17.
Ready to submit your research? Choose BMC and benefit from:

- fast, convenient online submission

- thorough peer review by experienced researchers in your field

- rapid publication on acceptance

- support for research data, including large and complex data types

- gold Open Access which fosters wider collaboration and increased citations

- maximum visibility for your research: over $100 \mathrm{M}$ website views per year

At BMC, research is always in progress.

Learn more biomedcentral.com/submissions 